\title{
ON ESTIMATES FOR OSCILLATORY INTEGRALS WITH PHASE DEPENDING ON PARAMETERS
}

\author{
SH.A. MURANOV
}

\begin{abstract}
We consider estimates for the Fourier transforms of measures supported on analytic hypersurfaces involving a damping factor. As a damper, we naturally take a power of the Gaussian curvature of the surface. It is known that if the exponent in this power is a sufficiently large positive number, then the Fourier transform of the corresponding measure has an optimal decay. C.D. Sogge and E.M. Stein formulated a problem on a minimal power of the Gaussian curvature ensuring an optimal decay for the Fourier transform. In the paper we resolve the problem by C.D. Sogge and E.M. Stein on an optimal decay for the Fourier transform with a damping factor for a particular class of families of analytic surfaces in the three-dimensional Euclidean space. We note that the power we provide is sharp not only for the families of analytic hypersurfaces but also for a fixed analytic hypersurface. The proof of main result is based on the methods of the theory of analytic functions, more precisely, on the statements like a preparation Weierstrass theorem. As D.M. Oberlin showed, similar statements fail for infinitely differentiable hypersurfaces.
\end{abstract}

Keywords: oscillating integrals, Fourier transform, dumping factor, maximal operator.

Mathematics Subject Classification: 35D05; 35D10; 35G05

\section{INTRODUCTION}

In connection with the problem on bounding maximal operators associated with a hypersurface $S \subset \mathbb{R}^{n+1}$, S.D. Sogge and I.M. Stein [1] introduced the following damped oscillating integrals:

$$
\widehat{\mu}_{q}(\xi):=\int_{S} e^{i(\xi, x)}|K(x)|^{q} \psi(x) d \sigma(x),
$$

where $K(x)$ is the Gaussian curvature of the hypersurface at a point $x \in S, \psi \in C_{0}^{\infty}(S)$ is a smooth non-negative compactly supported function, $(x, \xi)$ is a scalar product of the vectors $x$ and $\xi, d \sigma(x)$ is the surface measure. They showed that if $q \geqslant 2 n$, then integral (1.1) decays as $O\left(|\xi|^{-\frac{n}{2}}\right)$ as $|\xi| \rightarrow+\infty$, that is, it decays optimally. We observe that if the Gaussian curvature does not vanish, the Fourier transform of the surface measure decays as $O\left(|\xi|^{-\frac{n}{2}}\right)$ as $|\xi| \rightarrow+\infty$ and for a non-zero measure, it can not decay faster and this indicates the optimality of the decay rate. For a family of smooth hypersurfaces $S(\eta) \subset \mathbb{R}^{n+1}$ smoothly depending on parameters $\eta \in \mathbb{R}^{m}$, the measure $d \mu(\eta):=\psi(x, \eta) d \sigma(x, \eta)$ is naturally defined as well as the following oscillating integrals with a damping factor:

$$
\widehat{\mu}_{q}(\xi)=\int_{S(\eta)} e^{i(x, \xi)}|K(x, \eta)|^{q} \psi(x, \eta) d \sigma(x, \eta),
$$

where for each fixed $\eta$ and $d \sigma(x, \eta)$ is the surface measure $S(\eta)$.

Sh.A. Muranov, On estimates for oscillatory integrals With Phase DePending On Parameters.

(c) Muranov Sh.A. 2019.

The work is supported by Committee of Education, Science and Innovative Activity of the Ministry of Higher and Special Education of the Republic of Uzbekistan (grant no. OT-F4-69).

Submitted October 8, 2018. 
Formulation of problem Find a minimal $q$ ensuring the following estimate:

$$
\left|\widehat{\mu}_{q}(\xi)\right| \leqslant A|\xi|^{-\frac{n}{2}} \text {. }
$$

A similar problem for a fixed hypersurface $S$ was posed in work [1] by Sogge and Stein. The solution for the formulated problem in one-dimensional case, namely, as $S$ is a curve defined by a polynomial, follows the results by D. Oberlin [2]. In fact, the results by D. Oberlin are related with a family of curves.

In the present work we provide a solution to the problem of S.D. Sogge and I.M. Stein for a particular class of analytic surfaces in a three-dimensional space depending on parameters.

A function $\left.(x, \tau)\right|_{S_{(\eta)}}$, where $\tau \in S^{2}$, that is, $\tau$ is an arbitrary vector belonging to the unit sphere centered at the origin, is the restriction of the family of functions $(x, \tau)$ depending on $\tau$ and $\eta$ on a surface $S(\eta) \subset \mathbb{R}^{3}$ called a phase function.

For instance, if $S=\left\{\left(x_{1}, x_{2}, \Psi\left(x_{1}, x_{2}, \eta\right)\right)\right\}$, where $\Psi\left(x_{1}, x_{2}, \eta\right)=x_{1}^{2}+x_{2}^{4}+\eta x_{2}^{2}$, then $\left.(x, \tau)\right|_{S_{(\eta)}}=\tau_{1} x_{1}+\tau_{2} x_{2}+\tau_{3} \Psi\left(x_{1}, x_{2}, \eta\right)$ is the phase function corresponding to $S$.

Let $y=\Phi(x), x \in \mathbb{R}^{n}$, be some function with a critical point $x=x^{0}$. If in some neighbourhood $\Omega\left(x^{0}\right)$ of the point $x^{0}$, there exists a diffeomorphic change $\varphi: \Sigma \mapsto \Omega\left(x^{0}\right)$, where $\Sigma \subset \mathbb{R}^{n}$ is a neighbourhood of the zero, the function $\Phi(x)$ is reduced to the form

$$
\Phi(\varphi(z))=\Phi\left(x^{0}\right) \pm z_{1}^{k+1} \pm z_{2}^{2} \pm z_{3}^{2} \pm \cdots \pm z_{n}^{2},
$$

then $x=x^{0}$ is called a critical point of type $A_{k}[3$.

The next theorem was proved in work [4], see also [5].

Theorem 1.1. Let $q \geqslant 1$ be a fixed real number and $S(\eta) \subset \mathbb{R}^{3}$ be a family of analytic hypersurfaces depending on a parameter $\eta \in \mathbb{R}^{m}$. If the phase function corresponding to the hypersurface $S(0)$ has a singularity of type $A_{k}, 1 \leqslant k<\infty$, at the point $(0,0,0) \in S(0)$, then there exists a neighbourhood of the zero $V \times U \subset \mathbb{R}^{3} \times \mathbb{R}^{m}$ such that for each function $\psi \in C_{0}^{\infty}(V \times U)$, integral 1.2 satisfies the following estimate:

$$
\left|\widehat{\mu}_{q}(\xi)\right| \leqslant \frac{C\|\psi(\cdot, \eta)\|_{C^{2}}}{|\xi|}
$$

where $C$ is fixed positive number.

The main result of the present work is the following theorem.

Theorem 1.2. Let $q \geqslant 1$ be a fixed real number, $S(\eta) \subset \mathbb{R}^{3}$ be a family of analytic hypersurfaces satisfying the following conditions:

1. The hypersurface $S(0)$ contains the origin in $\mathbb{R}^{3}$ and at least one of the principal curvatures of the surfaces $S(0)$ is non-zero at the origin.

2. The Gaussian curvature $K(x, \eta)$ on the hypersurface $S(\eta)$ obeys the condition $K \not \equiv 0$.

Then there exists a neighbourhood of the origin $V \times U \subset \mathbb{R}^{3} \times \mathbb{R}^{m}$ such that for each function $\psi \in C_{0}^{\infty}(V \times U)$ integral $(1.2)$ satisfies the following estimate:

$$
\left|\widehat{\mu}_{q}(\xi)\right| \leqslant \frac{C\|\psi(\cdot, \eta)\|_{C^{2}}}{|\xi|}
$$

where $C$ is a fixed positive number.

\section{AuXiLiary STATEMENTS}

According the assumptions of Theorem 1.2, we can suppose that $\psi(x, \eta)$ has a sufficiently small support. Moreover, we assume that $S(\eta)$ is a graph of some analytic function $x_{3}=$ $f\left(x_{1}, x_{2}, \eta\right)$ defined in a small neighbourhood of the origin:

$$
S(\eta):=\left\{\left(x_{1}, x_{2}\right) \in V_{1} \subset \mathbb{R}^{2}: x_{3}=f\left(x_{1}, x_{2}, \eta\right), \eta \in U\right\},
$$

and

$$
f(0,0,0)=0, \quad \nabla_{x} f(0,0,0)=0 .
$$


Indeed, let $S(\eta)$ be a family of analytic hypersurfaces depending on $\eta \in U \subset \mathbb{R}^{m}$. Then, after a possible motion, we can assume that $S(0)$ contains the origin and the tangential plane $T_{0} S(0)$ at the origin is given by the equation $x_{3}=0$.

This is why, in the vicinity of the point $(0,0,0)$, the surface $S(0)$ is described by the equation $F\left(x_{1}, x_{2}, x_{3}\right)=0$, where $F$ is a real-analytic function satisfying the conditions

$$
F(0,0,0)=0, \quad \frac{\partial F(0,0,0)}{\partial x_{1}}=\frac{\partial F(0,0,0)}{\partial x_{2}}=0 \quad \text { and } \quad \frac{\partial F(0,0,0)}{\partial x_{3}} \neq 0 .
$$

According the implicit function theorem, in the vicinity of zero, the equation $F\left(x_{1}, x_{2}, x_{3}\right)=0$ possesses an analytic solution $x_{3}=\Phi\left(x_{1}, x_{2}\right)$. Hence, $\Phi\left(x_{1}, x_{2}\right)$ is an analytic function obeying the conditions $\Phi(0,0)=0, \nabla \Phi(0,0)=0$. In the same way, for the family $S(\eta)$, there exists a function $f\left(x_{1}, x_{2}, \eta\right)$ such that in the vicinity of zero, the surface $S(\eta)$ is defined by the equation $x_{3}=f\left(x_{1}, x_{2}, \eta\right)$ and $f\left(x_{1}, x_{2}, \eta\right)$ satisfies the condition $f\left(x_{1}, x_{2}, 0\right)=\Phi\left(x_{1}, x_{2}\right)$; for more details see [6].

We note that the function $(x, \tau)$ has no stationary points as $\tau \neq 0$ since $(x, \tau)_{x}=\tau$. But its restriction on $S$ has stationary (critical), see [7, Ch. III, Sect. 4]. These are the points $x(\tau)$, at which the hypersurface $(x, \tau)=$ const touches $S$.

Lemma 2.1. A stationary point $x(\tau) \in S$ is non-degenerate if and only if the Gaussian curvature of the hypersurface $S$ at this point is non-zero.

This lemma was proved in [7, Ch. III, Sect. 4].

We note that if the Gaussian curvature obeys $K(0,0,0) \neq 0$, then according Lemma 2.1, in a small neighbourhood of the point $(0,0,0)$, the phase function $\left.(x, \tau)\right|_{S(\eta)}$ has only non-degenerate critical points since if the Gaussian curvature is non-zero in the neighbourhood of zero $V \times U$, then $|K(x, \eta)|{ }^{q} \psi(x, \eta) \in C_{0}^{\infty}(V \times U)$. This is why, according Morse lemma, see [7], it is reduced to the sum of squares and the integral $\widehat{\mu}_{q}(\xi)$ satisfies the relation: $\widehat{\mu}_{q}(\xi)=O\left(|\xi|^{-1}\right)$ as $|\xi| \rightarrow \infty$. Therefore, in this case, Theorem 1.2 holds true. In what follows we assume that $K(0,0,0)=0$.

Before we proceed to proving Theorem 1.2, we consider some needed auxiliary statements.

Lemma 2.2. Let $g=g(x)$ be a real continuously differentiable function defined on $[c, d]$. If for $(x, \eta) \in[c, d] \times U$ the inequality $\left|g^{\prime}\right| \geqslant \delta>0$ holds and the functions $a(\cdot, \eta), g^{\prime}(\cdot, \eta)$ have $a$ bounded variation on $[c, d]$, then the following estimate holds:

$$
\left|\int_{c}^{d} e^{i \lambda g(x, \eta)} a(x, \eta) d x\right| \leqslant \frac{C\left\|\frac{a(\cdot, \eta)}{g^{\prime}}\right\|_{V}}{|\lambda|}
$$

where $\|a(\cdot, \eta)\|_{V}:=|a(c, \eta)|+V_{c}^{d}[a(\cdot, \eta)]$ and $V_{c}^{d}[a(\cdot, \eta)]$ is a total variation of the function a on $[c, d]$.

Proof. First we write integral as

$$
\int_{c}^{d} e^{i \lambda g(x, \eta)} a(x, \eta) d x=\int_{c}^{d} \frac{a(x, \eta)}{i \lambda g^{\prime}(x, \eta)} d\left(e^{i \lambda g(x, \eta)}\right) .
$$

Employing the integration by parts for the Stieltjes integral, we obtain the estimate

$$
\left|\int_{c}^{d} e^{i \lambda g(x, \eta)} a(x, \eta) d x\right| \leqslant\left|\frac{a(d, \eta)}{i \lambda g^{\prime}(d, \eta)} e^{i \lambda g(d, \eta)}-\frac{a(c, \eta)}{i \lambda g^{\prime}(c, \eta)} e^{i \lambda g(c, \eta)}\right|+\left|\frac{1}{i \lambda} \int_{c}^{d} e^{i \lambda g(x, \eta)} d\left(\frac{a(x, \eta)}{g^{\prime}(x, \eta)}\right)\right| .
$$

Finally, we observe that $\max _{x \in[c, d]}|a(x, \eta)| \leqslant\|a(\cdot, \eta)\|_{V}$ and this is why, if $a(x, \eta)$ and $g^{\prime}(x, \eta)$ are the functions with a bounded variation, we arrive at estimate (2.1).

Lemma 2.2 is an analogue of Statement II in Proposition 2 in monograph [8, see also 9], [10].

In this work we employ the following technical lemmata proved in work [11]: 
Lemma 2.3. Let a function $f \not \equiv 0$ be real-analytic at zero $\mathbb{R} \times \mathbb{R}^{m}$ such that $f(0,0)=0$. There exists a real-analytic manifold $Y$ and a mapping $\pi: Y \mapsto \mathbb{R}^{m}$ being proper such that for each point $y^{0} \in Y$ there exists a chart $\left(\varphi_{1}, \ldots, \varphi_{m}\right)$ centered at the point $y^{0}$, for which the relation

$$
f\left(x_{2}, \pi(y)\right)=\varphi_{1}^{\alpha_{1}}(y) \varphi_{2}^{\alpha_{2}}(y) \ldots \varphi_{m}^{\alpha_{m}}(y) b\left(x_{2}, y\right) p\left(x_{2}, y\right)
$$

holds, where $b\left(x_{2}, y\right), b\left(0, y^{0}\right) \neq 0$, is a real-analytic function, $p\left(x_{2}, y\right)$ is an unitary pseudopolynomial, that is,

$$
p\left(x_{2}, y\right)=x_{2}^{m_{1}}+\tau_{1}(y) x_{2}^{m_{1}-1}+\tau_{2}(y) x_{2}^{m_{1}-2}+\cdots+\tau_{m_{1}}(y) .
$$

Here functions $\tau_{1}, \ldots, \tau_{m_{1}}$ are real-analytic at the point $y^{0}$ and $\tau_{\ell}\left(y^{0}\right)=0, \ell=1, \ldots, m_{1}$.

Lemma 2.4. Let a function $f:\left(\mathbb{R} \times \mathbb{R}^{m}, 0\right) \mapsto(\mathbb{R}, 0)$ be real-analytic at the origin. There exists a neighbourhood of the zero $W \times U \subset \mathbb{R} \times \mathbb{R}^{m}$ such that for each fixed positive number $q$, the function $|f(\cdot, \eta)|^{q}$ has a bounded variation in $W$ and a total variation of this function $V_{W}\left[|f(\cdot, \eta)|^{q}\right]$ is a bounded function in $U$.

We shall also make use of the following lemma.

Lemma 2.5. Let a function $f(x, \eta)$ be real-analytic function at the origin and $q \geqslant 1$ be a fixed number. Then there exists a neighbourhood of the zero $W \times U$ in $\mathbb{R} \times \mathbb{R}^{m}$, in which the identity

$$
|x| g(x, \eta)=|f(x, \eta)|^{q}-|f(0, \eta)|^{q},
$$

holds, where the function $g(x, \eta)$ has a bounded variation in $W$ and its total variation is bounded in $U$.

Proof. In fact, Lemma 2.5 is an analogue of Lemma 3.3 in work [11]. For the reader's convenience, we provide here a detailed proof of this lemma.

We first assume that $f(x, \eta)$ is a polynomial. We assume that

$$
f(x, \eta):=Q(x, \eta)=x^{\ell}+\eta_{1} x^{\ell-1}+\cdots+\eta_{\ell}
$$

and the coefficients of the polynomial are bounded; $|\eta| \leqslant 1$.

We are going to show that the function

$$
g(x, \eta)=\frac{|Q(x, \eta)|^{q}-\left|\eta_{\ell}\right|^{q}}{|x|}
$$

has a bounded variation over the segment $[-1,1]$ and its total variation $V_{-1}^{1}[g(\cdot, \eta)]$ is bounded by a constant depending only on $\ell$ and $q$. It is easy to show that $g(x, \eta)$ is a piece-wise monotone function. Indeed, let $x>0$ and $Q(x, \eta)>0$. Then the numerator and the denominator are differentiable. We calculate the derivative of the function $g(x, \eta)$ in $x$ and we obtain

$$
g^{\prime}(x, \eta)=\frac{x q(Q(x, \eta))^{q-1} Q^{\prime}(x, \eta)-\left((Q(x, \eta))^{q}-\left|\eta_{\ell}\right|^{q}\right)}{x^{2}} .
$$

Let us show that the numerator has at most $2 \ell$ zeroes. We calculate the derivative of the numerator and equate it at zero and we obtain:

$$
q x(Q(x, \eta))^{q-2}\left((q-1)\left(Q^{\prime}(x, \eta)\right)^{2}+Q(x, \eta) Q^{\prime \prime}(x, \eta)\right)=0 .
$$

The latter equation has at most $2 \ell-2$ zeroes since $\left.Q^{\prime}(x, \eta)\right)^{2}+Q(x, \eta) Q^{\prime \prime}(x, \eta)$ is a polynomial of degree $2 \ell-2$.

This is why the numerator can have at most $2 \ell-2$ zeroes as $Q>0$. In the same way we consider the case $Q(x, \eta)<0$ and $x<0$. This implies that equation $g^{\prime}(x, \eta)=0$ has at most $4 \ell-4$ zeroes.

Since $q \geqslant 1$, then for $x, y \in[-1,1]$ we have an obvious inequality:

$$
\|\left. x\right|^{q}-|y|^{q}|\leqslant C(q)| x-y \mid,
$$


where $C(q)$ is some positive number depending only on $q \geqslant 1$. This implies that the function $g(x, \eta)$ is bounded by the number $C(q) \frac{\ell(\ell-1)}{2}$ as $|x| \leqslant 1$. Indeed,

$$
\left|x \left\|g(x, \eta)\left|\leqslant\left\|\left.Q(x, \eta)\right|^{q}-|Q(0, \eta)|^{q}|\leqslant C(q)| Q(x, \eta)-Q(0, \eta)\left|\leqslant C(q) \max _{-1 \leqslant \zeta \leqslant 1}\right| Q^{\prime}(\zeta, \eta)\right\| x\right|\right.\right.
$$

and hence,

$$
\max _{-1 \leqslant x \leqslant 1}|g(x, \eta)| \leqslant C(q) \max _{-1 \leqslant \zeta \leqslant 1}\left|Q^{\prime}(\zeta, \eta)\right| \leqslant C(q) \frac{\ell(\ell-1)}{2} .
$$

Then we have

$$
V_{-1}^{1}[g(x, \eta)] \leqslant(4 \ell-4) \max _{[-1,1]}|g(x, \eta)| \leqslant C(q) 2 \ell(\ell-1)^{2} .
$$

Thus, the total variation of the function $g$ over the segment $[-1,1]$ is estimated by a constant depending only on $\ell$ and $q$.

Let $f(x, \eta)$ be an arbitrary real-analytic function. In this case, employing Lemma 2.3, we reduce out function to the form

$$
f(x, \pi(y))=\varphi_{1}^{\alpha_{1}}(y) \varphi_{2}^{\alpha_{2}}(y) \ldots \varphi_{m}^{\alpha_{m}}(y) b(x, y) Q(x, y),
$$

where $b$ is a real-analytic function satisfying the condition $b(0,0) \neq 0$ and $Q(x, y)$ is some pseudo-polynomial. In this case we get the relation

$$
\begin{aligned}
|b(x, y) Q(x, y)|^{q}-|b(0, y) Q(0, y)|^{q}= & |Q(x, y)|^{q}\left(|b(x, y)|^{q}-|b(0, y)|^{q}\right) \\
& +|b(0, y)|^{q}\left(|Q(x, y)|^{q}-|Q(0, y)|^{q}\right) .
\end{aligned}
$$

We observe that the function $\frac{|b(x, y)|^{q}-|b(0, y)|^{q}}{|x|}$ has a bounded variation since $b(0,0) \neq 0$. And also, according Lemma 2.4 the function $|Q(x, y)|^{q}$ has a bounded variation in the coordinate neighbourhood $V$ as $q \geqslant 1$.

Finally, we observe that $\pi: Y \mapsto U$ is a proper analytic mapping [13]. This is why $\pi^{-1}(\bar{U}) \subset Y$ is a compact set. Therefore, for each point $y^{0} \in \pi^{-1}(\bar{U})$ we can find a coordinate neighbourhood $V \subset Y$ of the point $y^{0}$ such that as $y \in V$, we have the relation

$$
f(x, \pi(y))=\varphi_{1}^{\alpha_{1}}(y) \varphi_{2}^{\alpha_{2}}(y) \ldots \varphi_{m}^{\alpha_{m}}(y) b(x, y) Q(x, y),
$$

where $\left(\varphi_{1}, \ldots, \varphi_{m}\right)$ are local coordinates centered at $y^{0}$, that is, $\varphi_{j}\left(y^{0}\right)=0, j=1, \ldots, m$, $b(x, y)$ is a real-analytic function satisfying the condition $b(x, y) \neq 0$ as $(x, y) \in W \times V$, and $Q(x, y)$ is a pseudo-polynomial and $\alpha_{j} \geqslant 0, j=1, \ldots, m$, are integer numbers.

According the proven facts, the function $f(x, \pi(y))$ satisfies the assumptions of Lemma 2.5 in the vicinity $W \times V$. Since $\pi^{-1}(\bar{U})$ is a compact set, we can choose a finite covering $\pi^{-1}(\bar{U})$ and a neighbourhood of the zero $W \subset \mathbb{R}$ such that the statement of Lemma 2.5 is true for $(x, y) \in$ $W \times \pi^{-1}(\bar{U})$. This is why, the statement of Lemma 2.5 holds on the set $W \times U \subset \mathbb{R} \times \mathbb{R}^{m}$. This completes the proof of Lemma 2.5 .

We provide an analogue of Erdélyi lemma [14].

Lemma 2.6. If $F(x, s)$ is a smooth function defined in a small neighbourhood of the origin $W \times U \in \mathbb{R} \times \mathbb{R}^{m}$ and satisfying the conditions

$$
F^{\prime}(0, s)=0, \quad F^{\prime \prime}(0, s) \neq 0 \quad \text { for each } \quad s \in U \quad \text { and } \quad a \in C_{0}^{\infty}(W \times U),
$$

then for $0 \leqslant q \leqslant 1$, the inequality holds:

$$
\left.\left|\int_{-\varepsilon}^{\varepsilon}\right| x\right|^{q} e^{i \lambda F(x, s)} a(x, s) d x \mid \leqslant \frac{C_{q}\|a(\cdot, s)\|_{V}}{|\lambda|^{\frac{q+1}{2}}},
$$

where $\varepsilon$ is a sufficiently small positive number. 
Proof. We employ Morse lemma with parameters, see [7]. According this lemma, there exists a diffeomorphism $x=x(y, s)$ mapping the segment $I=[-\varepsilon, \varepsilon]$ into $\left[-\delta_{1}(\varepsilon), \delta_{2}(\varepsilon)\right]$ such that the function $F(x, s)$ reads as $F(x(y, s), s)=F(0, s) \pm y^{2}$ and $x(0, s) \equiv 0$. It follows from the latter identity that $x(y, s)$ is written as $x(y, s)=y G(y, s)$ with a smooth function $G(y, s)$ and $G(0,0) \neq 0$.

We make the change of the variables $x=x(y, s)$ in the integral

$$
\int_{-\varepsilon}^{\varepsilon}|x|^{q} e^{i \lambda F(x, s)} a(x, s) d x
$$

and we obtain:

$$
I_{q}(\lambda)=e^{i \lambda F(0, s)} \int_{-\delta_{1}(\varepsilon)}^{\delta_{2}(\varepsilon)}|y|^{q} e^{ \pm i \lambda y^{2}} a_{1}(y, s) d y
$$

where

$$
a_{1}(y, s)=|G(y, s)|^{q}\left(G(y, s)+y G^{\prime}(y, s)\right) a(y G(y, s), s)
$$

and $a_{1}(y, s) \in C_{0}^{\infty}\left(\left[-\delta_{1}(\varepsilon), \delta_{2}(\varepsilon)\right]\right)$.

We proceed to estimating the integral $I_{q}(\lambda)$, which is written as $I_{q}(\lambda)=I_{1}(\lambda)+I_{2}(\lambda)$ for $0 \leqslant q \leqslant 1$. First we shall estimate the integral

$$
I_{1}(\lambda):=\int_{0}^{\delta_{2}(\varepsilon)} y^{q} e^{ \pm i \lambda y^{2}} a_{1}(y, s) d y,
$$

and then the estimate for the integral

$$
I_{2}(\lambda):=\int_{-\delta_{1}(\varepsilon)}^{0} y^{q} e^{ \pm i \lambda y^{2}} a_{1}(y, s) d y
$$

can be obtained in the same way.

If $\delta_{2}(\varepsilon) \leqslant \lambda^{-\frac{1}{2}}$ then by a trivial estimate for the integral we get

$$
\left|I_{1}(\lambda)\right| \leqslant \frac{\max _{y \in\left[0, \delta_{2}(\varepsilon)\right]}\left|a_{1}(y, s)\right|}{\lambda^{\frac{q+1}{2}}} .
$$

Now we assume that $\delta_{2}(\varepsilon)>\lambda^{-\frac{1}{2}}$. In this case the integral $I_{1}(\lambda)$ is written as a sum of the following two integrals:

$$
I_{11}(\lambda)=\int_{0}^{\lambda^{-\frac{1}{2}}} y^{q} e^{ \pm i \lambda y^{2}} a_{1}(y, s) d y \quad \text { and } \quad I_{12}(\lambda)=\int_{\lambda^{-\frac{1}{2}}}^{\delta_{2}(\varepsilon)} y^{q} e^{ \pm i \lambda y^{2}} a_{1}(y, s) d y .
$$

It is obvious that $I_{11}(\lambda)$ obeys an estimate of form $(2.3)$.

We integrate by parts in the integral $I_{12}(\lambda)$ and we obtain the following estimate:

$$
\left|I_{12}(\lambda)\right| \leqslant \lambda^{-\frac{q+1}{2}} C_{1} V_{0}^{\delta_{2}}\left[a_{1}(\cdot, s)\right] .
$$

Then, by means of inequalities (2.3) and (2.4), we get the estimate

$$
\left|I_{1}(\lambda)\right| \leqslant \frac{C\left\|a_{1}(\cdot, s)\right\|_{V}}{\lambda^{\frac{q+1}{2}}}, \quad C=\text { const. }
$$

Summing up the obtained estimate, we complete the proof.

Lemma 2.7. There exists a neighbourhood $V_{1} \times U \subset \mathbb{R}^{2} \times \mathbb{R}^{m}$ of the origin such that for each $q>0, \psi \in C_{0}^{\infty}\left(V_{1} \times U\right)$ and $\max \left\{\left|\xi_{1}\right|,\left|\xi_{2}\right|\right\} \geqslant\left|\xi_{3}\right|$, the following estimate holds:

$$
\left|\widehat{\mu}_{q}(\xi)\right| \leqslant \frac{C\|\psi\|_{C^{1}}}{|\xi|}
$$


Proof. We observe that

$$
K\left(x_{1}, x_{2}, \eta\right)=\frac{\operatorname{Hess} f\left(x_{1}, x_{2}, \eta\right)}{\left(1+\left|\nabla f\left(x_{1}, x_{2}, \eta\right)\right|^{2}\right)^{2}}
$$

is an analytic function in a small neighbourhood of the origin, see [6]. Since $\left|\nabla_{x} f(0,0,0)\right|=0$, there exists a neighbourhood of the origin $V_{1} \times U$ such that for each point $\left(x_{1}, x_{2}, \eta\right) \in V_{1} \times U$, the inequality holds: $\left|\nabla_{x} f\left(x_{1}, x_{2}, \eta\right)\right| \leqslant \frac{1}{2}$. Without loss of generality we can assume that $\left|\xi_{1}\right|=\max \left\{\left|\xi_{1}\right|,\left|\xi_{2}\right|\right\} \geqslant\left|\xi_{3}\right|$. The case $\left|\xi_{2}\right|=\max \left\{\left|\xi_{1}\right|,\left|\xi_{2}\right|\right\} \geqslant\left|\xi_{3}\right|$ can be considered in the same way. In this case the integral $\widehat{\mu}_{q}(\xi)$ is written as the following two-dimensional damped oscillating integral:

$$
\widehat{\mu}_{q}(\xi)=\int_{\mathbb{R}^{2}} e^{i\left(x_{1} \xi_{1}+x_{2} \xi_{2}+f\left(x_{1}, x_{2}, \eta\right) \xi_{3}\right)} a\left(x_{1}, x_{2}, \eta\right)\left|\operatorname{Hess} f\left(x_{1}, x_{2}, \eta\right)\right|{ }^{q} d x_{1} d x_{2},
$$

where

$$
a\left(x_{1}, x_{2}, \eta\right)=\frac{\psi\left(x_{1}, x_{2}, f\left(x_{1}, x_{2}, \eta\right)\right)}{\sqrt{\left(1+\left|\nabla f\left(x_{1}, x_{2}, \eta\right)\right|^{2}\right)^{4 q-1}}} .
$$

We employ the Fubini theorem for integral (2.5) and we obtain:

$$
\widehat{\mu}_{q}(\xi)=\int_{\mathbb{R}} \widehat{\mu}_{q}^{0}\left(\xi_{1}, \xi_{3}, x_{2}\right) e^{i \xi_{3} s_{2} x_{2}} d x_{2}
$$

where

$$
\begin{aligned}
& \widehat{\mu}_{q}^{0}\left(\xi_{1}, \xi_{3}, x_{2}\right)=\int_{\mathbb{R}} e^{i \xi_{1} F_{1}\left(x_{1}, x_{2}, \xi_{1}, \xi_{3}, \eta\right)} a\left(x_{1}, x_{2}, \eta\right)\left|\operatorname{Hess} f\left(x_{1}, x_{2}, \eta\right)\right|{ }^{q} d x_{1}, \\
& F_{1}\left(x_{1}, x_{2}, \xi_{1}, \xi_{3}, \eta\right)=\frac{\xi_{3}}{\xi_{1}} f\left(x_{1}, x_{2}, \eta\right)+x_{1} .
\end{aligned}
$$

It is obvious that for each $\left(x_{1}, x_{2}, \eta\right) \in V_{1} \times U$, the inequality holds:

$$
\left|F_{1 x_{1}}^{\prime}\left(x_{1}, x_{2}, \xi_{1}, \xi_{2}, \eta\right)\right|=\left|1+\frac{\xi_{3}}{\xi_{1}} f_{x_{1}}^{\prime}\left(x_{1}, x_{2}, \eta\right)\right| \geqslant \frac{1}{2} .
$$

According Lemma 2.4, the function $\left|\operatorname{Hess} f\left(x_{1}, x_{2}, \eta\right)\right|^{q}$ has a bounded variation in $\operatorname{Pr}_{1}\left(V_{1}\right)$ and its total variation is bounded in $\operatorname{Pr}_{2}\left(V_{1}\right) \times U$ for each $q>0$, where $\operatorname{Pr}_{1}\left(V_{1}\right)\left(\operatorname{Pr}_{2}\left(V_{1}\right)\right)$ is its projection on the axis $\mathbb{R}_{x_{1}}\left(\mathbb{R}_{x_{2}}\right)$. This is why, employing Lemma 2.6, we obtain the following inequality:

$$
\left|\widehat{\mu}_{q}^{0}\left(\xi_{1}, \xi_{3}, x_{2}\right)\right| \leqslant \frac{C_{1}\left\|a\left(\cdot, x_{2}, \eta\right)\right\|_{C^{1}}}{\left|\xi_{1}\right|} \leqslant \frac{\sqrt{3} C_{1}\left\|a\left(\cdot, x_{2}, \eta\right)\right\|_{C^{1}}}{|\xi|} .
$$

Integrating the latter inequality over $\operatorname{Pr}_{2}\left(V_{1}\right)$, for the integral $\widehat{\mu}_{q}(\xi)$ we find the estimate:

$$
\left|\widehat{\mu}_{q}(\xi)\right| \leqslant \frac{C\|\psi\|_{C^{1}}}{|\xi|}
$$

This completes the proof.

Corollary 1. Let $\varepsilon>0$ be an arbitrary fixed positive number and $\Gamma_{\varepsilon} \in \mathbb{R}^{3}$ be the cone

$$
\Gamma_{\varepsilon}:=\left\{\xi \in \mathbb{R}^{3}: \varepsilon\left|\xi_{3}\right| \leqslant \max \left\{\left|\xi_{1}\right|,\left|\xi_{3}\right|\right\}\right\} .
$$

There exist a neighbourhood $V_{1} \times U$ of the origin and a positive number $C_{\varepsilon}>0$ such that for each $q>0, \psi \in C_{0}^{\infty}\left(V_{1} \times U\right)$ and $\xi \in \Gamma_{\varepsilon}$ the following estimate holds:

$$
\left|\widehat{\mu}_{q}(\xi)\right| \leqslant \frac{C_{\varepsilon}\|\psi\|_{C^{1}}}{|\xi|} .
$$

Corollary 1 shows that $\widehat{\mu}_{q}(\xi)$ satisfies the desired estimate as $\xi \in \Gamma_{\varepsilon}$ for each $q>0$.

We proceed to studying the behavior of $\widehat{\mu}_{q}(\xi)$ as $\xi \in \mathbb{R}^{3} \backslash \Gamma_{\varepsilon}$. 


\section{Asymptotic Behavior of $\widehat{\mu}_{q}(\xi)$}

In this section we study the behavior of $\widehat{\mu}_{q}(\xi)$ in the case $\xi \in \mathbb{R}^{3} \backslash \Gamma_{\varepsilon}$, where $\varepsilon$ is a sufficiently small fixed positive number. In this case $\widehat{\mu}_{q}(\xi)$ is written as the following two-dimensional damped oscillating integral:

$$
\widehat{\mu}_{q}(\xi)=\int_{\mathbb{R}^{2}} e^{i \xi_{3} F\left(x_{1}, x_{2}, s_{1}, s_{2}, \eta\right)} a\left(x_{1}, x_{2}, \eta\right)\left|\operatorname{Hess} f\left(x_{1}, x_{2}, \eta\right)\right|^{q} d x_{1} d x_{2},
$$

where

$$
\begin{aligned}
& a\left(x_{1}, x_{2}, \eta\right)=\frac{\psi\left(x_{1}, x_{2}, f\left(x_{1}, x_{2}, \eta\right)\right)}{\sqrt{\left(1+\left|\nabla f\left(x_{1}, x_{2}, \eta\right)\right|^{2}\right)^{4 q-1}}}, \\
& F\left(x_{1}, x_{2}, s_{1}, s_{2}, \eta\right)=f\left(x_{1}, x_{2}, \eta\right)+s_{1} x_{1}+s_{2} x_{2}, \quad s_{1}=\frac{\xi_{1}}{\xi_{3}}, \quad s_{2}=\frac{\xi_{2}}{\xi_{3}},
\end{aligned}
$$

Hess $f\left(x_{1}, x_{2}, \eta\right)=\operatorname{det} D^{2} f\left(x_{1}, x_{2}, \eta\right)$.

Choosing a small neighbourhood $V_{1} \times U$, we can assume that $\psi$ is an infinitely differentiable function with a sufficiently small support.

We observe that if $\operatorname{rank}\left(D^{2} f(0,0,0)\right)=1$, then one of the following inequalities holds:

$$
\frac{\partial^{2} f(0,0,0)}{\partial x_{1}^{2}} \neq 0 \quad \text { or } \quad \frac{\partial^{2} f(0,0,0)}{\partial x_{2}^{2}} \neq 0 .
$$

For the sake of definiteness, we can assume that

$$
\frac{\partial^{2} f(0,0,0)}{\partial x_{1}^{2}} \neq 0
$$

Then according the implicit function theorem, the equation

$$
F_{x_{1}}\left(x_{1}, x_{2}, s_{1}, s_{2}, \eta\right)=f_{x_{1}}\left(x_{1}, x_{2}, \eta\right)+s_{1}=0
$$

has a unique analytic solution $x_{1}=x_{1}\left(x_{2}, s_{1}, \eta\right)$ in a small neighbourhood of the origin in $\mathbb{R}_{x_{1}} \times \mathbb{R}_{s_{1}} \times U$.

According the Fubini theorem, integral (3.1) is written as

$$
\widehat{\mu}_{q}(\xi)=\int_{\mathbb{R}} \widehat{\mu}_{q}^{1}\left(\xi, x_{2}\right) e^{i \xi_{3} s_{2} x_{2}} d x_{2},
$$

where

$$
\widehat{\mu}_{q}^{1}\left(\xi, x_{2}\right)=\int_{\mathbb{R}} e^{i \xi_{3} F_{1}\left(x_{1}, x_{2}, s_{1}, \eta\right)} a\left(x_{1}, x_{2}, \eta\right)\left|\operatorname{Hess} f\left(x_{1}, x_{2}, \eta\right)\right|^{q} d x_{1}
$$

and

Now we consider integral $\widehat{\mu}_{q}^{1}\left(\xi, x_{2}\right)$.

$$
F_{1}\left(x_{1}, x_{2}, s_{1}, \eta\right)=f\left(x_{1}, x_{2}, \eta\right)+s_{1} x_{1} .
$$

Proposition 1. If an analytic function $f\left(x_{1}, x_{2}, \eta\right)$ satisfies the conditions

$$
\nabla f(0,0,0)=0, \quad \frac{\partial^{2} f(0,0,0)}{\partial x_{1}^{2}} \neq 0,
$$

then there exists a neighbourhood $V_{1} \times U \subset \mathbb{R}^{2} \times \mathbb{R}^{m}$ of the origin such that as $q \geqslant 1$, the integral $\widehat{\mu}_{q}^{1}$ satisfies the following asymptotic relation:

$$
\begin{aligned}
\widehat{\mu}_{q}^{1}\left(\xi, x_{2}\right)= & \sqrt{\frac{2 \pi}{\left|\xi_{3}\right|}} e^{i\left(\frac{\pi}{4} \operatorname{sgn}\left(\frac{\partial^{2} f(0,0,0)}{\partial x_{1}^{2}} \xi_{3}\right)+\xi_{3} F_{1}\left(x_{1}\left(x_{2}, s_{1}, \eta\right), x_{2}, s_{1}\right)\right)} \\
& \cdot\left|\operatorname{Hess} f\left(x_{1}\left(x_{2}, s_{1}, \eta\right), x_{2}, \eta\right)\right|^{q} a\left(x_{2}, s_{1}, \eta\right)+O\left(\frac{1}{|\xi|}\right) \quad \text { as } \quad|\xi| \rightarrow+\infty
\end{aligned}
$$


where $a\left(x_{2}, s_{1}, \eta\right):=a\left(x_{1}\left(x_{2}, s_{1}, \eta\right), x_{2}, \eta\right) \phi\left(x_{2}, s_{1}, \eta\right)$. Here $\phi$ is some smooth function being $O\left(\frac{1}{|\xi|}\right)$ uniformly in small $\left(x_{2}, s_{1}, \eta\right)$, that is, there exists $C>0$ and a neighbourhood $U_{1}$ of the zero such that for all $\left(x_{2}, s_{1}, \eta\right) \in U_{1}$, the inequality

$$
\left|O\left(\frac{1}{|\xi|}\right)\right| \leqslant \frac{C}{|\xi|}
$$

holds.

Proof. In the integral $\widehat{\mu}_{q}^{1}$, we change the variables

$$
x_{1}=X_{1}+x_{1}\left(x_{2}, s_{1}, \eta\right)
$$

and we obtain:

$$
\begin{aligned}
\widehat{\mu}_{q}^{1}\left(\xi, x_{2}\right)= & \int_{\mathbb{R}} e^{i \xi_{3} F_{1}\left(X_{1}+x_{1}\left(x_{2}, s_{1}, \eta\right), x_{2}, s_{1}, \eta\right)} a\left(X_{1}+x_{1}\left(x_{2}, s_{1}, \eta\right), x_{2}, \eta\right) \\
& \cdot\left|\operatorname{Hess} f\left(X_{1}+x_{1}\left(x_{2}, s_{1}, \eta\right), x_{2}, \eta\right)\right|^{q} d X_{1} .
\end{aligned}
$$

We observe that Hess $f\left(X_{1}+x_{1}\left(x_{2}, s_{1}, \eta\right), x_{2}, \eta\right)$ is a real-analytic function and since $q \geqslant 1$, it follows from Lemma 2.5 that

$$
\left|\operatorname{Hess} f\left(X_{1}+x_{1}\left(x_{2}, s_{1}, \eta\right), x_{2}\right)\right|^{q}-\left|\operatorname{Hess}\left(x_{1}\left(x_{2}, s_{1}, \eta\right), x_{2}\right)\right|^{q}=\left|X_{1}\right| \vartheta\left(X_{1}, x_{2}, s_{1}, \eta\right),
$$

where $\vartheta\left(X_{1}, x_{2}, s_{1}, \eta\right)$ is a function with a bounded variation on $X_{1}$ and its total variation $V_{-\delta}^{\delta}\left[\vartheta\left(\cdot, x_{2}, s_{1}, \eta\right)\right]$ is a bounded function on $\left(x_{2}, s_{1}, \eta\right)$.

We write integral $\widehat{\mu}_{q}^{1}\left(\xi, x_{2}\right)$ as

$$
\begin{aligned}
\widehat{\mu}_{q}^{1}\left(\xi, x_{2}\right)= & \int_{\mathbb{R}} e^{i \xi_{3} F_{1}\left(X_{1}+x_{1}\left(x_{2}, s_{1}, \eta\right), x_{2}, s_{1}, \eta\right)} a\left(X_{1}+x_{1}\left(x_{2}, s_{1}, \eta\right), x_{2}, \eta\right) \\
& \cdot\left[\left|\operatorname{Hess} f\left(X_{1}+x_{1}\left(x_{2}, s_{1}, \eta\right), x_{2}, \eta\right)\right|^{q}-\left|\operatorname{Hess} f\left(x_{1}\left(x_{2}, s_{1}, \eta\right), x_{2}, \eta\right)\right|^{q}\right] d X_{1} \\
& +\left|\operatorname{Hess} f\left(x_{1}\left(x_{2}, s_{1}, \eta\right), x_{2}, \eta\right)\right|^{q} \int_{\mathbb{R}} e^{i \xi_{3} F_{1}\left(X_{1}+x_{1}\left(x_{2}, s_{1}, \eta\right), x_{2}, s_{1}, \eta\right)} \\
& \cdot a\left(X_{1}+x_{1}\left(x_{2}, s_{1}, \eta\right), x_{2}, \eta\right) d X_{1}=I_{1}+I_{2} .
\end{aligned}
$$

Employing relation 3.2 , we can write $I_{1}$ as an oscillating integral with a damping factor:

$$
I_{1}=\int_{\mathbb{R}} e^{i \xi_{3} F_{1}\left(X_{1}+x_{1}\left(x_{2}, s_{1}, \eta\right), x_{2}, s_{1}, \eta\right)}\left|X_{1}\right| a_{1}\left(X_{1}, x_{2}, s_{1}, \eta\right) d X_{1},
$$

where

$$
a_{1}\left(X_{1}, x_{2}, s_{1}, \eta\right)=a\left(X_{1}+x_{1}\left(x_{2}, s_{1}, \eta\right), x_{2}, \eta\right) \vartheta\left(X_{1}, x_{2}, s_{1}, \eta\right)
$$

and $\left|X_{1}\right|$ serves as a damping. According Lemma 2.6, we obtain the inequality:

$$
\left|I_{1}\right| \leqslant \frac{C\left\|a\left(\cdot, x_{2}, s_{1}, \eta\right) \cdot \vartheta\left(\cdot, x_{2}, s_{1}, \eta\right)\right\|_{V}}{\left|\xi_{3}\right|} \leqslant \frac{C_{1}\left\|a\left(\cdot, x_{2}, s_{1}, \eta\right) \cdot \vartheta\left(\cdot, x_{2}, s_{1}, \eta\right)\right\|_{V}}{|\xi|},
$$

since $\left|\xi_{3}\right| \geqslant \frac{1}{\sqrt{3}}|\xi|$ and $C_{1}=\sqrt{3} C$.

We consider the following one-dimensional oscillating integral:

$$
I_{2}=\left|\operatorname{Hess} f\left(x_{1}\left(x_{2}, s_{1}, \eta\right), x_{2}, \eta\right)\right|^{q} \int_{\mathbb{R}} e^{i \xi_{3} F_{1}\left(X_{1}+x_{1}\left(x_{2}, s_{1}, \eta\right), x_{2}, s_{1}, \eta\right)} a\left(X_{1}+x_{1}\left(x_{2}, s_{1}, \eta\right), x_{2}, \eta\right) d X_{1} .
$$

We note that the amplitude of the latter oscillating integral is a smooth function with a sufficiently small support.

Thanks to the Morse lemma, there exists a neighbourhood $\mathbb{R}_{y} \times \mathbb{R}_{x_{2}} \times \mathbb{R}_{s_{1}} \times U$ of the origin and a diffeomorphism

$$
\left(X_{1}, x_{2}, s_{1}, \eta\right) \mapsto\left(X_{1}\left(y, x_{2}, s_{1}, \eta\right), x_{2}, s_{1}, \eta\right)
$$


such that the phase function $F_{1}\left(X_{1}+x_{1}\left(x_{2}, s_{1}, \eta\right), x_{2}, s_{1}, \eta\right)$ is reduced to the form

$$
F_{1}\left(X_{1}\left(y, x_{2}, s_{1}, \eta\right)+x_{1}\left(x_{2}, s_{1}, \eta\right), x_{2}, s_{1}, \eta\right)= \pm y^{2}+F_{1}\left(x_{1}\left(x_{2}, s_{1}, \eta\right), x_{2}, s_{1}, \eta\right),
$$

and the sign at $y^{2}$ coincides with $\operatorname{sgn}\left(\frac{\partial^{2} f}{\partial x_{1}^{2}}(0,0,0)\right)$ and $\left(X_{1}(0, \cdot, \cdot, \cdot) \equiv 0\right.$. Hence, for the oscillating integral $I_{2}$ we have

$$
\begin{aligned}
I_{2}= & \left|\operatorname{Hess} f\left(x_{1}\left(x_{2}, s_{1}, \eta\right), x_{2}, \eta\right)\right|^{q} e^{i \xi_{3} F_{1}\left(x_{1}\left(x_{2}, s_{1}, \eta\right), x_{2}, s_{1}, \eta\right)} \\
& \cdot \int_{\mathbb{R}} e^{ \pm i \xi_{3} y^{2}} a\left(X_{1}\left(y, x_{2}, s_{1}, \eta\right)+x_{1}\left(x_{2}, s_{1}, \eta\right), x_{2}, \eta\right) \frac{\partial X_{1}\left(y, x_{2}, s_{1}, \eta\right)}{\partial y} d y .
\end{aligned}
$$

Now we employ the standard stationary phase method [7, to obtain

$$
\begin{aligned}
& I_{2}=\left|\operatorname{Hess} f\left(x_{1}\left(x_{2}, s_{1}, \eta\right), x_{2}, \eta\right)\right|^{q} e^{i \xi_{3} F_{1}\left(x_{1}\left(x_{2}, s_{1}, \eta\right), x_{2}, s_{1}, \eta\right)} \\
& \cdot\left(\sqrt{\frac{2 \pi}{\xi_{3}}} e^{ \pm i s g n\left(\xi_{3}\right) \frac{\pi}{4}} a\left(x_{2}, s_{1}, \eta\right)+O\left(\frac{1}{\left|\xi_{3}\right|^{\frac{3}{2}}}\right)\right),
\end{aligned}
$$

where

$$
a\left(x_{2}, s_{1}, \eta\right)=a\left(x_{1}\left(x_{2}, s_{1}, \eta\right), x_{2}, \eta\right) \frac{\partial X_{1}\left(0, x_{2}, s_{1}, \eta\right)}{\partial y} .
$$

As a result, for the oscillating integral $\widehat{\mu}_{q}^{1}\left(\xi, x_{2}\right)$ we have:

$$
\begin{aligned}
\widehat{\mu}_{q}^{1}\left(\xi, x_{2}\right)= & \sqrt{\frac{2 \pi}{\left|\xi_{3}\right|}} e^{i\left( \pm \frac{\pi}{4} \operatorname{sgn}\left(\xi_{3}\right)+\xi_{3} F_{1}\left(x_{1}\left(x_{2}, s_{1}, \eta\right), x_{2}, s_{1}, \eta\right)\right)} \\
& \cdot\left|\operatorname{Hess} f\left(x_{1}\left(x_{2}, s_{1}, \eta\right), x_{2}, \eta\right)\right|^{q} a\left(x_{2}, s_{1}, \eta\right)+O\left(\frac{1}{|\xi|}\right)
\end{aligned}
$$

where

$$
F_{1}\left(x_{1}\left(x_{2}, s_{1}, \eta\right), x_{2}, s_{1}, \eta\right)=s_{1} x_{1}\left(x_{2}, s_{1}, \eta\right)+f\left(x_{1}\left(x_{2}, s_{1}, \eta\right), x_{2}, \eta\right) .
$$

This completes the proof.

Corollary 2. Let $f\left(x_{1}, x_{2}, \eta\right)$ satisfies the assumptions of Proposition 1, then there exists a neighbourhood of the zero $V_{1} \times U \subset \mathbb{R}^{2} \times \mathbb{R}^{m}$ such that, as $q \geqslant 1$, the integral $\widehat{\mu}_{q}$ satisfies the asymptotic formula:

$$
\begin{aligned}
\widehat{\mu}_{q}(\xi)= & \sqrt{\frac{2 \pi}{\left|\xi_{3}\right|}} e^{\frac{\pi}{4} i \operatorname{sgn}\left(\frac{\partial^{2} f(0,0,0)}{\partial x_{1}^{2}} \xi_{3}\right)} \\
& \cdot \int e^{i \xi_{3}\left(F_{1}\left(x_{1}\left(x_{2}, s_{1}, \eta\right), x_{2}, s_{1}\right)+s_{2} x_{2}\right)}\left|\operatorname{Hessf}\left(x_{1}\left(x_{2}, s_{1}, \eta\right), x_{2}, \eta\right)\right|^{q} a\left(x_{2}, s_{1}, \eta\right) d x_{2} \\
& \left.+O\left(\frac{1}{|\xi|}\right) \text { as }|\xi| \rightarrow+\infty\right)
\end{aligned}
$$

where

$$
a\left(x_{2}, s_{1}, \eta\right):=a\left(x_{1}\left(x_{2}, s_{1}, \eta\right), x_{2}, \eta\right) \phi\left(x_{2}, s_{1}, \eta\right) .
$$

Here $\phi$ is some smooth function being $O\left(\frac{1}{|\xi|}\right)$ uniformly with respect to the small parameters $\eta$.

This corollary follows immediately Proposition 1 .

The next lemma is proved by straightforward calculations, see [15].

Lemma 3.1. Let $F\left(x_{1}, x_{2}, s_{1}, s_{2}, \eta\right)$ be a smooth function depending on the parameters $\left(s_{1}, s_{2}, \eta\right)$ and

$$
F_{x_{1}}^{\prime}(0,0,0,0,0)=0, \quad F_{x_{1}}^{\prime \prime}(0,0,0,0,0) \neq 0
$$


If $x_{1}=x_{1}\left(x_{2}, s_{1}, s_{2}, \eta\right)$ is a smooth solution of the equation $F_{x_{1}}^{\prime}\left(x_{1}, x_{2}, s_{1}, s_{2}, \eta\right)=0$, then the second derivative of the function $F\left(x_{1}\left(x_{2}, s_{1}, s_{2}, \eta\right), x_{2}, s_{1}, s_{2}, \eta\right)$ with respect to $x_{2}$ obeys the following identity:

$$
\frac{\partial^{2} F\left(x_{1}\left(x_{2}, s_{1}, s_{2}, \eta\right), x_{2}, s_{1}, s_{2}, \eta\right)}{\partial x_{2}^{2}}=\frac{\operatorname{Hess} F\left(x_{1}\left(x_{2}, s_{1}, s_{2}, \eta\right), x_{2}, s_{1}, s_{2}, \eta\right)}{\frac{\partial^{2} F\left(x_{1}\left(x_{2}, s_{1}, s_{2}, \eta\right), x_{2}, s_{1}, s_{2}, \eta\right)}{\partial x_{1}^{2}}} .
$$

Since Hess $F_{1}\left(x_{1}, x_{2}, s_{1}, \eta\right)=$ Hess $f\left(x_{1}, x_{2}, \eta\right)$, then by Lemma 3.1 we have:

$$
\frac{\partial^{2} F_{1}\left(x_{1}\left(x_{2}, s_{1}, \eta\right), x_{2}, s_{1}\right)}{\partial x_{2}^{2}}=\frac{\operatorname{Hess} f\left(x_{1}\left(x_{2}, s_{1}, \eta\right), x_{2}\right)}{\frac{\partial^{2} f\left(x_{1}\left(x_{2}, s_{1}, \eta\right), x_{2}, \eta\right)}{\partial x_{1}^{2}}}
$$

Hence, it is sufficient to consider the behavior of the following one-dimensional oscillating integral with the damping factor:

$$
I_{q}\left(\xi_{3}\right)=\int e^{i \xi_{3}\left(F_{1}\left(x_{2}, s_{1}, \eta\right)+x_{2} s_{2}\right)} \widetilde{a}\left(x_{2}, s_{1}, \eta\right)\left|F_{1}^{\prime \prime}\left(x_{2}, s_{1}, \eta\right)\right|^{q} d x_{2}
$$

where

$$
\widetilde{a}\left(x_{2}, s_{1}, \eta\right)=a\left(x_{2}, s_{1}, \eta\right)\left|\frac{\partial^{2}}{\partial x_{1}^{2}} f\left(x_{1}\left(x_{2}, s_{1}, \eta\right), x_{2}\right)\right|^{q}
$$

and $F_{1}\left(x_{2}, s_{1}, \eta\right)$ is an analytic function. If $F_{1}\left(x_{2}, s_{1}, \eta\right)$ is a polynomial, then by Oberliner theorem [2], as $q \geqslant \frac{1}{2}$, for integral (3.3) we obtain:

$$
I_{q}\left(\xi_{3}\right)=O\left(\frac{1}{\left|\xi_{3}\right|^{\frac{1}{2}}}\right) \quad \text { as } \quad\left|\xi_{3}\right| \rightarrow \infty .
$$

In what follows, we shall prove an analogue of Oberliner theorem for analytic functions; this theorem is of an independent interest. The proof of main theorem 1.2 is reduced to problem on estimating one-dimensional oscillating integrals with an arbitrary analytic phase depending on the parameters, which is a more general result. As D.M. Oberliner showed [2], a similar statement fails for the functions in the class $C^{\infty}$.

\section{Proof of Theorem 1.2}

Now we consider the following one-dimensional oscillating integral:

$$
I_{q}\left(\xi_{3}\right)=\int e^{i \xi_{3} F\left(x_{2}, \eta\right)} a\left(x_{2}, \eta\right)\left|F^{\prime \prime}\left(x_{2}, \eta\right)\right|^{q} d x_{2},
$$

where $F\left(x_{2}, \eta\right)$ is real-analytic function in the vicinity of zero $W \times U \subset \mathbb{R} \times \mathbb{R}^{m}$ satisfying the conditions $F\left(x_{2}, \eta\right) \not \equiv 0, F(0,0)=0$ and $a\left(x_{2}, \eta\right) \in C_{0}^{\infty}(W \times U)$.

We begin with an auxiliary lemma.

Lemma 4.1. Let $F\left(x_{2}, \eta\right)$ a function real-analytic on the set $W \times \pi^{-1}(\bar{U})$. Then for each point $y^{0} \in \pi^{-1}(\bar{U})$ there exists a neighbourhood $\omega \subset \mathbb{R}^{m}$ such that for each $q \geqslant \frac{1}{2}$, the integral $I_{q}\left(\xi_{3}\right)$ satisfies the following estimate

$$
\left|I_{q}\left(\xi_{3}\right)\right| \leqslant \frac{C\|a(\cdot, y)\|_{V}}{\left|\xi_{3}\right|^{\frac{1}{2}}}
$$

where $\omega \subset \mathbb{R}^{m}$ is a corresponding neighbourhood of the origin in $\mathbb{R}^{m}$.

Proof. In the proof, we employ a covering of the set $\pi^{-1}(\bar{U})$ by finitely many neighbourhoods $\omega_{j}$ of the points $y^{j} \in \pi^{-1}(\bar{U})$.

Let $y^{0}$ be some fixed point in $\pi^{-1}(\bar{U})$. Since $\varphi^{\alpha}\left(\varphi^{\alpha}=\varphi_{1}^{\alpha_{1}}(y) \varphi_{2}^{\alpha_{2}}(y) \ldots \varphi_{m}^{\alpha_{m}}(y)\right)$ is bounded in some neighbourhood of the point $y^{0}$, the function $F_{1}\left(x_{2}, y\right)$ reads as

$$
F_{1}\left(x_{2}, y\right)=\varphi^{\alpha} F_{2}\left(x_{2}, y\right)
$$

where $F_{2}\left(x_{2}, y\right)=b\left(x_{2}, y\right) p\left(x_{2}, y\right)$. 
It follows from Lemma 2.3 that for each point $y^{0} \in Y$ of this manifold there exists a coordinate neighbourhood $\omega$ such that $F_{2}\left(x_{2}, y\right)$ is a function real-analytic at the point $\left(0, y^{0}\right)$ and the condition holds $F_{2}\left(x_{2}, y^{0}\right) \not \equiv 0$. This is why we can apply a Weierstrass preparation theorem to the function $F_{2}\left(x_{2}, y\right)$.

We note that $\varphi^{\alpha}$ is bounded in the neighbourhood $\omega$. Employing Proposition 2.1 in work [4] and applying standard methods for the analysis for $q \geqslant \frac{1}{2}$, we arrive at the following estimate:

$$
\left|I_{q}\left(\xi_{3}\right)\right| \leqslant \frac{C}{\left|\xi_{3}\right|^{\frac{1}{2}}} .
$$

Since $\pi^{-1}(\bar{U})$ is a compact set, reproducing the above arguing for each set $\omega_{j}$, we get estimate (4.2). Finally, these local estimates allow us to obtain the desired estimate for $\pi^{-1}(\bar{U})$. The proof is complete.

Now we are going to prove the following proposition.

Proposition 2. Let $F\left(x_{2}, \eta\right)$ be afunction real-analytic at zero. Then there exists a neighbourhood $W \times U \subset \mathbb{R} \times \mathbb{R}^{m}$ of the origin such that for each real number $q \geqslant \frac{1}{2}$, the following estimate holds true:

$$
\left|I_{q}\left(\xi_{3}\right)\right| \leqslant \frac{C\|a\|_{V}}{\left|\xi_{3}\right|^{\frac{1}{2}}} .
$$

Proof. If the function $F\left(x_{2}, \eta\right)$ satisfies the assumptions of the Weierstrass preparation theorem [16], that is, $F\left(x_{2}, 0\right) \not \equiv 0$, then we obtain the result of work [4] since in this case the function $F\left(x_{2}, 0\right)$ has a singularity of type $A_{k}$. This condition is equivalent to the following: at the point $x_{2}=0$, the function $F_{x_{2}}^{\prime}\left(x_{2}, 0\right)$ has a root of multiplicity $k<\infty$ under the condition $F_{x_{2}}^{\prime}(0,0)=0$.

We consider the case, when the function $F\left(x_{2}, \eta\right)$ does not satisfy the assumptions of the Weierstrass preparation theorem [16]. More precisely, we consider the case, when $F\left(x_{2}, 0\right) \equiv 0$ although $F \not \equiv 0$. We note that as it was shown in the classical work by W. Osgood [17, Sect. 2], no analogues of Weierstrass theorem holds in this case.

In the case, when $F\left(x_{2}, \eta\right)$ is analytically continued to the set $\mathrm{C} \times B$, where $B \subset \mathrm{C}^{m}$ is some ball centered at the origin, we can employ Lemma 3 of work [12]. However, as the Osgood counterexample indicates [17, Sect. 2], in the general case, Lemma 3 in work [12] fails. However, we can employ Lemma 2.3 since $F\left(x_{2}, \eta\right)$ is a non-zero real analytic function on the set $W \times U$ and $F(0,0)=0$. Then, applying Lemma 2.3 , we construct a manifold $Y$ and a proper analytic mapping $\pi: Y \mapsto U$ such that in local coordinates, the function $F\left(x_{2}, \pi(y)\right)$ reads as

$$
F\left(x_{2}, \pi(y)\right)=\varphi_{1}^{\alpha_{1}}(y) \varphi_{2}^{\alpha_{2}}(y) \ldots \varphi_{m}^{\alpha_{m}}(y) b\left(x_{2}, y\right) p\left(x_{2}, y\right)
$$

where $p\left(x_{2}, y\right)$ is a pseudo-polynomial and $\varphi(y)$ are local coordinates. In this case, for each point $y^{0} \in Y$ there exist local coordinates $\left(\varphi_{1}, \ldots, \varphi_{m}\right)$ centered at this point and obeying the conditions $\varphi_{j}\left(y^{0}\right)=0, j=1, \ldots, m$. We suppose that $\pi^{-1}(\bar{U}) \subset Y$ is some compact set on a real-analytic manifold $Y$.

Therefore, integral (4.1) reads as

$$
I_{q}\left(\xi_{3}\right)=\int e^{i \xi_{3} F_{1}\left(x_{2}, y\right)} a\left(x_{2}, y\right)\left|F_{1}^{\prime \prime}\left(x_{2}, y\right)\right|^{q} d x_{2},
$$

where

$$
F_{1}\left(x_{2}, y\right)=\varphi_{1}^{\alpha_{1}}(y) \varphi_{2}^{\alpha_{2}}(y) \ldots \varphi_{m}^{\alpha_{m}}(y) b\left(x_{2}, y\right) p\left(x_{2}, y\right)
$$

Since $\pi: Y \mapsto U$ is a proper analytic mapping [13], we apply Lemma 4.1] and standard methods from the analysis and this proves Proposition 2. Indeed, according Lemma 4.1, for each point $y^{0} \in \pi^{-1}(\bar{U})$ there exists a coordinate neighbourhood $V$ centered at the point $y^{0}$ such that $F\left(x_{2}, \pi(y)\right)$ is written as

$$
F\left(x_{2}, \pi(y)\right)=\varphi_{1}^{\alpha_{1}}(y) \varphi_{2}^{\alpha_{2}}(y) \ldots \varphi_{m}^{\alpha_{m}}(y) b\left(x_{2}, y\right) p\left(x_{2}, y\right)
$$


where $b\left(x_{2}, y\right)$ is a real-analytic function and $b\left(x_{2}, y\right) \neq 0$ for each point $\left(x_{2}, y\right) \in W \times \omega$ and $p\left(x_{2}, y\right)$ is unitary pseudo-polynomial.

Thus, we have the needed estimate as $\left(x_{2}, y\right) \in W \times \omega$. Since $\pi^{-1}(\bar{U})$ is a compact set, there exists a finite covering $\pi^{-1}(\bar{U}) \subset \bigcup_{j=1}^{N} \omega_{j}$ and neighbourhoods $W_{j}$ of the zero such that in $W_{j} \times \omega_{j}$ we have the desired estimate. Finally, redenoting $W:=\bigcap_{j=1}^{N} W_{j} \neq \varnothing$, we get the desired estimate in $W \times U$ and this completes the proof.

Proposition 2 completes the proof of Theorem 1.2 .

In conclusion, the author expresses his deep gratitude to the referee for valuable remarks.

\section{BIBLIOGRAPHY}

1. C.D. Sogge, E.M. Stein. Averages of functions over hypersurfaces in $\mathbb{R}^{n} / /$ Invent. Math. 82:3, 543-556 (1985).

2. D.M. Oberlin. Oscillatory integrals with polynomial phase // Math. Scand. 69:1, 45-56 (1991).

3. V.I. Arnold, S.M. Gusein-Zade, A.N. Varchenko. Singularities of differentiable maps, Volume 1. Classification of critical points, caustics and wave fronts. Nauka, Moscow (1982). [Modern Birkhäuser Classics. Birkhäuser, Boston (2012).]

4. Sh.A. Muranov. On estimates for oscillatory integrals with damping factor // Uzbek Math. J. 4, $112-125$ (2018).

5. I.A. Ikromov, Sh.A. Muranov. Estimates of oscillatory integrals with a damping factor // Matem. Zamet. 104:2, 236-251 (2018). [Math. Notes, 104:2 218-230 (2018).]

6. B.A. Dubrovin, A.T. Fomenko, S.P. Novikov. Modern geometry - methods and applications. Part I. The geometry of surfaces, transformation groups, and fields. Editorial URSS, Moscow (1998). [Graduate Texts in Mathematics. 93. Springer-Verlag, New York etc.]

7. M.V. Fedoryuk. Saddle-point method. Nauka, Moscow (1977). (in Russian).

8. E.M. Stein. Harmonic analysis: real-variable methods, orthogonality, and oscillatory integrals. Princeton University Press, Princeton (1993).

9. G.I. Arkhipov, A.A. Karatsuba and V.N. Chubarikov. Trigonometric integrals // Izv. Akad. Nauk SSSR Ser. Mat. 43:5, 971-1003 (1979). [Math. USSR-Izv. 15:2, 211-239 (1980).]

10. J.G. VanDer Corput. Zahlentheoretische Abschätzungen // Math. Ann. 84, 53-79 (1921). (in German).

11. I.A. Ikromov. Damped oscillatory integrals and maximal operators // Matem. Zamet. 78:6, 833852 (2005). [Math. Notes. 78:6, 773-790 (2005).]

12. A.S. Sadullaev. Criteria for analytic sets to be algebraic // Funkts. Anal. Pril. 6:1, 85-86 (1972). [Funct. Anal. Appl. 6:1, 78-79 (1972).]

13. Ed. Bierstone, P.D. Milman. Arc-analytic functions // Invent. Math. 101, 411-424 (1990).

14. A. Erdélyi. Asymptotic expansions // Dover Publications, New York (1956).

15. I.A. Ikromov, D. Müller, M. Kempe. Damped oscillatory integrals and boundedness of maximal operators associated to mixed homogeneous hypersurfaces // Duke Math. J. 126:3, 471-490 (2005).

16. B. Malgrange. Ideals of differentiable functions. Oxford University Press, London (1966).

17. W. Osgood. Lehrbuch der Funktionentheorie. Bd. II. Teubner, Leipzig (1929). (in German).

Shkhriddin Abdullaevich Muranov,

Samarkand State University, Universitetskii boulevard 15, 140104, Samarkand, Uzbekistan

E-mail: muranov-2017@mail.ru 